\title{
The inner power of a graph
}

\author{
Richard H. Hammack \\ Department of Mathematics and Applied Mathematics \\ Virginia Commonwealth University, Richmond, VA 23284-2014, USA \\ Neal D. Livesay \\ Department of Mathematics, Louisiana State University \\ Baton Rouge, LA 70803-4918, USA
}

Received 2 June 2010, accepted 1 October 2010, published online 10 November 2010

\begin{abstract}
We define a new graph operation called the $k$ th inner power. The construction-which is somewhat analogous to the $k$ th power with respect to the direct product-seems to lend itself nicely to certain questions concerning cancellation over the direct product. We prove several results about bipartiteness and connectedness of inner powers, and we prove that the inner power distributes over the direct product. We explore a potential connection between inner powers and the problem of cancellation over the direct product.
\end{abstract}

Keywords: Graph producs, graph operations, cancellation in graph products.

Math. Subj. Class.: 05C76, 05C99

\section{Introduction}

Our purpose is to define a new graph operation which we call an inner power. The article is organized as follows. The introduction recalls some relevant notation and lexicon; the second section defines the inner power and proves some of its elementary properties. In the final section we explore potential applications of the inner power to the problem of cancellation over the direct product.

We recall some standard definitions to fix the notation. Any graph $G=(V(G), E(G))$ in this paper is finite and may have loops, but not multiple edges. Edges are denoted $x y$, where $x, y \in V(G)$. A loop at $x$ is denoted $x x$. The cycle $C_{n}$ is the graph with vertices $0,1, \ldots, n-1$, where $i$ is adjacent to $i+1$ (with arithmetic done modulo $n$ ). The path $P_{n}$ has vertex set $1, \ldots, n$, with $i$ adjacent to $i+1$ for $1 \leq i<n$. We use the notation $G \subseteq H$

E-mail addresses: rhammack@vcu.edu (Richard H. Hammack), nlives1@1su.edu (Neal D. Livesay) 
to indicate that $G$ is a subgraph of $H$. A walk in $G$ is a sequence of vertices $x_{1} x_{2} \ldots x_{n}$ in $G$ for which $x_{i} x_{i+1} \in E(G)$ for each $1 \leq i<n$. The walk is closed if $x_{1}=x_{n}$. A homomorphism from a graph $G$ to a graph $H$ is a map $\varphi: V(G) \rightarrow V(H)$ for which $x y \in E(G)$ implies $\varphi(x) \varphi(y) \in E(H)$.

The direct product $G \times H$ is the graph with vertex set $V(G) \times V(H)$ and for which $(x, y)\left(x^{\prime}, y^{\prime}\right)$ is an edge precisely if $x x^{\prime} \in E(G)$ and $y y^{\prime} \in E(H)$. Figure 1 shows a direct product $G \times H$. For clarity, the factors $G$ and $H$ are drawn below and to the left of the product.

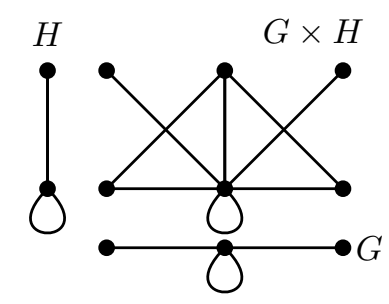

Figure 1: A direct product $G \times H$

The direct product can also be defined on $k$ factors. Given graphs $G_{1}, G_{2}, \ldots, G_{k}$, the product $G_{1} \times G_{2} \times \cdots \times G_{k}$ has as vertices the $k$-tuples $\left(x_{1}, x_{2}, \ldots, x_{k}\right)$, with $x_{i} \in V\left(G_{i}\right)$ for $1 \leq i \leq k$. Vertices $\left(x_{1}, x_{2}, \ldots, x_{k}\right)$ and $\left(y_{1}, y_{2}, \ldots, y_{k}\right)$ are adjacent provided that $x_{i} y_{i} \in E\left(G_{i}\right)$ for $1 \leq i \leq k$. The $k$ th direct power of a graph $G$ is the product $G^{k}=$ $G \times G \times \cdots \times G$ of $G$ with itself $k$ times.

See Imrich and Klavžar [2] for a survey of the direct product.

\section{The inner power}

We now introduce our main construction. Given a graph $G$, and a positive integer $k$, the $k$ th inner power of $G$ is the graph $G^{(k)}$ defined as follows, where arithmetic on the indices is done modulo $k$.

$$
\begin{aligned}
V\left(G^{(k)}\right) & =\left\{\left(x_{0}, x_{1}, \ldots, x_{k-1}\right): x_{i} \in V(G) \text { for } 0 \leq i<k\right\} \\
E\left(G^{(k)}\right) & =\left\{\left(x_{0}, x_{1}, \ldots, x_{k-1}\right)\left(y_{0}, y_{1}, \ldots, y_{k-1}\right): x_{i} y_{i \pm 1} \in E(G) \text { for } 0 \leq i<k\right\}
\end{aligned}
$$

Notice that if $k=2$, then $(x, y)\left(x^{\prime}, y^{\prime}\right)$ is an edge of $G^{(2)}$ if and only if $x y^{\prime} \in E(G)$ and $y x^{\prime} \in E(G)$. Figure 2 shows two examples. The left-hand side shows $K_{2}^{(2)}$, while the right-hand side shows the second inner power of the graph $G$ consisting of two loops.

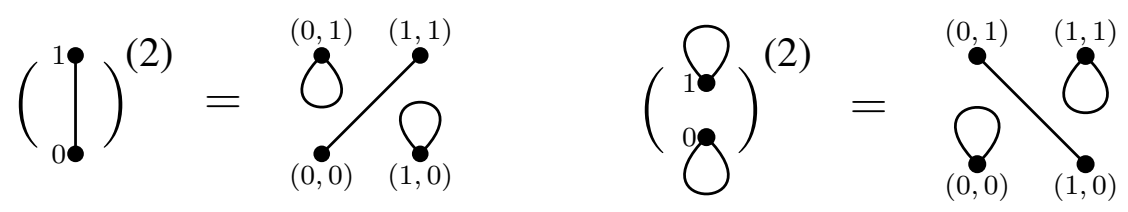

Figure 2: The inner powers $K_{2}^{(2)}$ and $G^{(2)}$, where $G$ consists of two loops. 
Figure 3 shows some more elaborate examples, namely $P_{3}^{(2)}$ and $C_{3}^{(2)}$, where, for convenience, the vertices are labeled as $x y$ rather than $(x, y)$. This figure illustrates a general principle that follows immediately from the definition of the inner power: If $G \subseteq H$, then $G^{(k)} \subseteq H^{(k)}$. Figure 4 shows the inner power $C_{3}^{(3)}$. Notice that in general the diagonal map $x \mapsto(x, x, \ldots, x)$ is an embedding of $G$ into $G^{(k)}$.
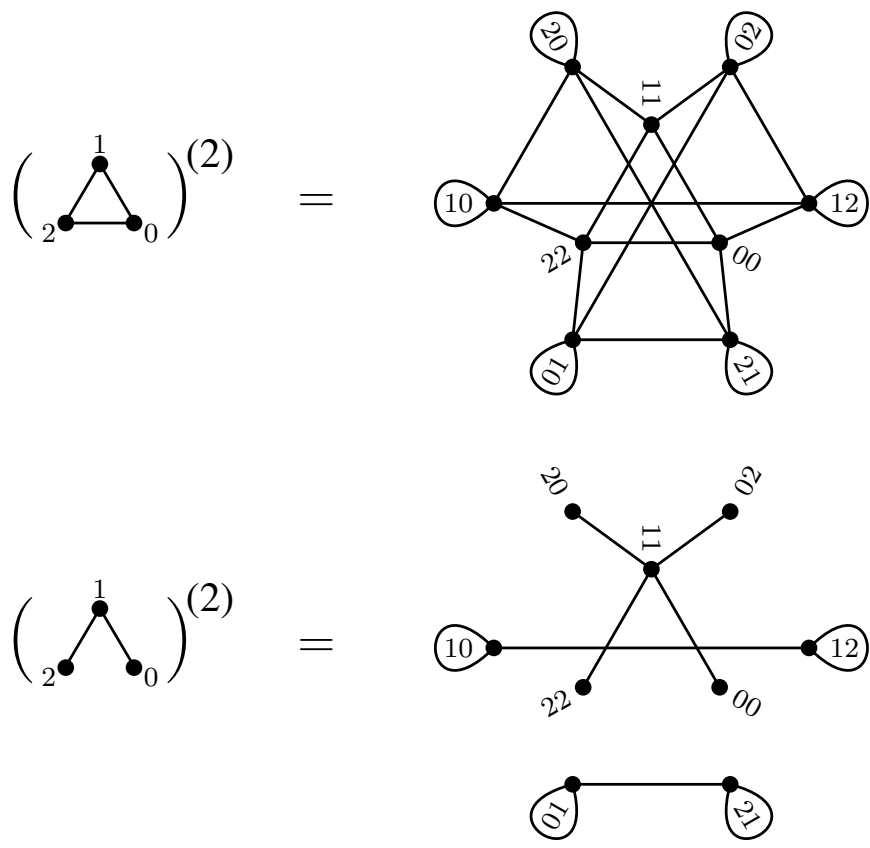

Figure 3: The inner powers $C_{3}^{(2)}$ and $P_{3}^{(2)}$. Note $G \subseteq H$ implies $G^{(k)} \subseteq H^{(k)}$.

We note in passing that $C_{3}^{(2)}$ and $C_{3}^{(3)}$ happen to coincide with the coloring graphs $\mathcal{C}_{3}\left(K_{2}\right)$ and $\mathcal{C}_{3}\left(C_{3}\right)$ (see Section 8.2 of [2]), suggesting a possible (and entirely unexplored) connection to graph colorings.

We now collect some properties of inner powers, beginning with a criterion that characterizes the presence of a loop in the inner power. The proof is an immediate consequence of the definitions, and is therefore omitted.

Proposition 2.1. The inner power $G^{(k)}$ has a loop at $\left(x_{0}, x_{1}, \ldots, x_{k-1}\right)$ if and only if the sequence $x_{0} x_{1} \ldots x_{k-1} x_{0}$ is a closed walk in $G$.

Thus if $k$ is even and $G$ has an edge $x y$, then $G^{(k)}$ has a loop at $(x, y, x, y, \ldots, x, y)$. Consequently an even inner power of a nontrivial graph is never bipartite. But we do have the following.

Proposition 2.2. An inner power $G^{(k)}$ is bipartite if and only if $G$ is bipartite and $k$ is odd.

Proof. If $G^{(k)}$ has no odd cycles, then as $G$ embeds in $G^{(k)}$, it follows that $G$ has no odd cycles. 

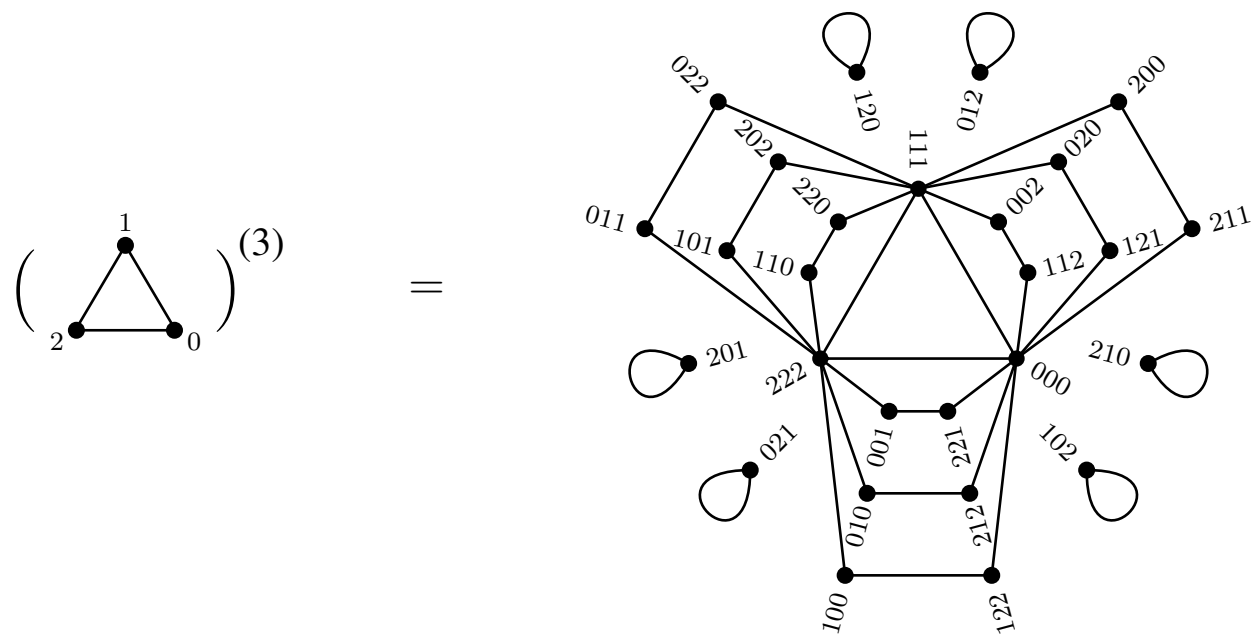

Figure 4: The inner power $C_{3}^{(3)}$.

Conversely, suppose $k$ is an odd integer and $G$ is a bipartite graph with partite sets $X$ and $Y$. Consider an arbitrary edge $\left(x_{0}, x_{1}, \ldots, x_{k-1}\right)\left(y_{0}, y_{1}, \ldots, y_{k-1}\right) \in E\left(G^{(k)}\right)$. Suppose, without loss of generality, that $x_{0} \in X$. Then since $x_{0} y_{1} \in E(G)$, we have $y_{1} \in Y$. In turn, since $y_{1} x_{2} \in E(G)$, we have $x_{2} \in X$. Similarly, $y_{3} \in Y, x_{4} \in$ $X$, and so on. Because $k$ is odd we infer $\left(x_{0}, x_{1}, \ldots, x_{k-1}\right) \in X \times X \times \cdots \times X$ and $\left(y_{0}, y_{1}, \ldots, y_{k-1}\right) \in Y \times Y \times \cdots \times Y$. It follows that any edge of $G^{(k)}$ has one endpoint in the set $X \times X \times \cdots \times X$ and the other endpoint in $V\left(G^{(k)}\right) \backslash X \times X \times \cdots \times X$. Thus $G^{(k)}$ is bipartite.

Recall (Theorem 5.5 of [2]) that a direct product $G \times H$ of nontrivial graphs is connected if and only if both $G$ and $H$ are connected and one of them has an odd cycle. Consequently a direct power $G^{k}$ is connected if and only if $G$ is connected and non-bipartite. By contrast, Figure 4 shows that inner powers of connected non-bipartite graphs may be disconnected. However (as the next result indicates) for $k=2$ the criteria for connectedness of the inner power is exactly the same as for the direct power.

Proposition 2.3. An inner power $G^{(2)}$ is connected if and only if $G$ is connected and has an odd cycle.

Proof. Suppose $G^{(2)}$ is connected. Then for any two vertices $x, y \in E(G)$, there is a path from $(x, y)$ to $(y, y)$ in $G^{(2)}$. Denote this path as

$$
(x, y)\left(x_{1}, y_{1}\right)\left(x_{2}, y_{2}\right)\left(x_{3}, y_{3}\right) \ldots\left(x_{n}, y_{n}\right)(y, y) .
$$

By the definition of the inner power, $x x_{1} y_{2} x_{3} y_{4} \ldots y$ is a walk in $G$ from $x$ to $y$. Thus $G$ is connected.

Conversely, suppose that $G$ is connected and contains an odd cycle. Let $(a, b),(c, d) \in$ $V\left(G^{(2)}\right)$. Then $G$ has an even walk from $a$ to $c$. (By traversing an odd cycle, if necessary.) 
Similarly, $G$ has an even walk from $b$ to $d$. What is more, we may assume these walks have the same length, by alternating back and forth along a final edge, if necessary. Therefore $G$ has walks $a x_{0} x_{1} x_{2} \ldots x_{2 n} c$ and $b y_{0} y_{1} y_{2} \ldots y_{2 n} d$. This gives rise to the walk

$$
(a, b)\left(y_{0}, x_{0}\right)\left(x_{1}, y_{1}\right)\left(y_{2}, x_{2}\right)\left(x_{3}, y_{3}\right) \ldots\left(y_{2 n}, x_{2 n}\right)(c, d)
$$

in $G^{(2)}$. Thus $G^{(2)}$ is connected.

Connectedness for powers higher than 2 is much more subtle issue, as the next result indicates.

Proposition 2.4. Suppose $k>2$. Then there is a walk in $G^{(k)}$ joining $\left(x_{0}, x_{1}, \ldots, x_{k-1}\right)$ to $\left(y_{0}, y_{1}, \ldots, y_{k-1}\right)$ if and only if for some $n$ there is a homomorphism $\varphi: P_{n} \times C_{k} \rightarrow G$, where $\varphi(1, i)=x_{i}$ and $\varphi(n, i)=y_{i}$ for each index $i$.

Proof. Suppose there is such a homomorphism. By the homomorphism property of $\varphi$, as well as the definitions of the direct product and the inner power, it follows that for each $1 \leq \ell<n$ the vertex $(\varphi(\ell, 0), \varphi(\ell, 1), \ldots, \varphi(\ell, k-1))$ is adjacent to

$$
(\varphi(\ell+1,0), \varphi(\ell+1,1), \ldots, \varphi(\ell+1, k-1))
$$

in $G^{(k)}$. Therefore $G^{(k)}$ has a walk from $\left(x_{0}, x_{1}, \ldots, x_{k-1}\right)$ to $\left(y_{0}, y_{1}, \ldots, y_{k-1}\right)$.

Conversely, suppose $G^{(k)}$ has a path from $\left(x_{0}, x_{1}, \ldots, x_{k-1}\right)$ to $\left(y_{0}, y_{1}, \ldots, y_{k-1}\right)$. Write this path as

$$
\left(v_{0}^{1}, v_{1}^{1}, \ldots, v_{k-1}^{1}\right)\left(v_{0}^{2}, v_{1}^{2}, \ldots, v_{k-1}^{2}\right)\left(v_{0}^{3}, v_{1}^{3}, \ldots, v_{k-1}^{3}\right) \ldots\left(v_{0}^{n}, v_{1}^{n}, \ldots, v_{k-1}^{n}\right),
$$

where the left-most vertex is $\left(x_{0}, x_{1}, \ldots, x_{k-1}\right)$ and the right-most is $\left(y_{0}, y_{1}, \ldots, y_{k-1}\right)$. Define $\varphi(\ell, i)=v_{i}^{\ell}$. One checks easily that this is the required homomorphism.

Proposition 2.4 yields the following corollary.

Corollary 2.5. The inner power $G^{(k)}$ is connected if and only if for any two $k$-tuples $\left(x_{0}, x_{1}, \ldots, x_{k-1}\right)$ and $\left(y_{0}, y_{1}, \ldots, y_{k-1}\right)$ of vertices of $G$, there exists a homomorphism $\varphi: P_{n} \times C_{k} \rightarrow G$, where $\varphi(1, i)=x_{i}$ and $\varphi(n, i)=y_{i}$.

Finally, we prove that the inner power distributes over the direct product.

Proposition 2.6. For any graphs $G$ and $H$, it follows that $(G \times H)^{(k)} \cong G^{(k)} \times H^{(k)}$.

Proof. Consider the bijection $\varphi: V\left((G \times H)^{(k)}\right) \rightarrow V\left(G^{(k)} \times H^{(k)}\right)$, where

$$
\varphi\left(\left(\left(x_{0}, y_{0}\right),\left(x_{1}, y_{1}\right), \ldots,\left(x_{k-1}, y_{k-1}\right)\right)\right)=\left(\left(x_{0}, x_{1}, \ldots, x_{k-1}\right),\left(y_{0}, y_{1}, \ldots, y_{k-1}\right)\right) \text {. }
$$

This is an isomorphism because

$$
\begin{aligned}
& \left(\left(x_{0}, y_{0}\right), \ldots,\left(x_{k-1}, y_{k-1}\right)\right)\left(\left(u_{0}, v_{0}\right), \ldots,\left(u_{k-1}, v_{k-1}\right)\right) \in E\left((G \times H)^{(k)}\right), \\
\Longleftrightarrow & \left(x_{i}, y_{i}\right)\left(u_{i \pm 1}, v_{i \pm 1}\right) \in E(G \times H) \text { for each index } i, \\
\Longleftrightarrow \quad & x_{i} u_{i \pm 1} \in E(G) \text { and } y_{i} v_{i \pm 1} \in E(H) \text { for each index } i \\
\Longleftrightarrow \quad & \left(x_{0}, x_{1}, \ldots, x_{k-1}\right)\left(u_{0}, u_{1}, \ldots, u_{k-1}\right) \in E\left(G^{(k)}\right) \text { and } \\
& \left(y_{0}, y_{1}, \ldots, y_{k-1}\right)\left(v_{0}, v_{1}, \ldots, v_{k-1}\right) \in E\left(H^{(k)}\right) \\
\Longleftrightarrow & \left(\left(x_{0}, \ldots, x_{k-1}\right),\left(y_{0}, \ldots, y_{k-1}\right)\right)\left(\left(u_{0}, \ldots, u_{k-1}\right),\left(v_{0}, \ldots, v_{k-1}\right)\right) \in \\
& E\left(G^{(k)} \times H^{(k)}\right) .
\end{aligned}
$$




\section{Cancellation}

The cancellation problem for the direct product involves the determination of the conditions under which $G \times K \cong H \times K$ implies $G \cong H$. We now review some classic results involving cancellation, and we note a potential application of inner powers to cancellation.

Given graphs $G$ and $H$, denote by $\operatorname{hom}(G, H)$ the number of homomorphisms from $G$ to $H$. The following theorem by Lovász is proved in [3] and [1]. (Actually, the cited proofs are for digraphs, but the arguments work equally well for graphs.)

Theorem 3.1. If $G$ and $H$ are graphs, then $G \cong H$ if and only if $\operatorname{hom}(X, A)=\operatorname{hom}(X, B)$ for every graph $X$.

Lovász [3] also noted the easily-proved identity

$$
\operatorname{hom}(X, A \times B)=\operatorname{hom}(X, A) \cdot \operatorname{hom}(X, B) .
$$

From this he deduced the following corollaries. (The first one involves the direct power of a graph, not the inner power.)

Corollary 3.2. If $G^{k} \cong H^{k}$, then $G \cong H$.

Proof. Suppose $G^{k} \cong H^{k}$. Let $X$ be any graph. Then $\operatorname{hom}\left(X, G^{k}\right)=\operatorname{hom}\left(X, H^{k}\right)$, and Equation (3.1) yields $\operatorname{hom}(X, G)^{k}=\operatorname{hom}(X, H)^{k}$. Thus $\operatorname{hom}(X, G)=\operatorname{hom}(X, H)$, so $G \cong H$ by Theorem 3.1 .

Corollary 3.3. If $G \times K \cong H \times K$ and $K$ has a loop, then $G \cong H$.

Proof. Suppose $G \times K \cong H \times K$. For any graph $X \operatorname{hom}(X, G \times K)=\operatorname{hom}(X, H \times K)$, and Equation (3.1) yields

$$
\operatorname{hom}(X, G) \cdot \operatorname{hom}(X, K)=\operatorname{hom}(X, H) \cdot \operatorname{hom}(X, K) .
$$

Now, $\operatorname{hom}(X, K) \neq 0$ because the constant map sending $V(X)$ to a vertex with a loop is a homomorphism. Thus $\operatorname{hom}(X, G)=\operatorname{hom}(X, H)$, so $G \cong H$ by Theorem 3.1.

Lovász [3] also proves the following generalization of Corollary 3.3: If $G \times K \cong H \times K$ and $K$ has an odd cycle, then $G \cong H$. His proof, involving a theory of $k$-partite structures, is quite complex and (at least to our thinking) non-intuitive. In the final part of this paper we explore the possibility that the inner power may lead to a simpler proof.

To this end, we first remark that the analogue of Corollary 3.2 does not hold for inner powers. Indeed, Figure 2 shows that it is possible to have $G^{(k)} \cong H^{(k)}$ with $G \neq H$. However, despite considerable effort we have found no examples of this phenomenon when $k$ is odd, and we are led to the following conjecture.

Conjecture 3.4. If $k$ is odd and $G^{(k)} \cong H^{(k)}$, then $G \cong H$.

We conclude by showing how the truth of the conjecture could lead to simpler proof of Lovász's cancellation theorem.

Proposition 3.5. Suppose Conjecture 3.4 is true. If $G \times K \cong H \times K$, and $K$ has an odd cycle, then $G \cong K$. 
Proof. Suppose $G \times K \cong H \times K$, and $K$ has an odd cycle $x_{0} x_{1} x_{2} \ldots x_{k-1} x_{0}$ of length $k$. Then $(G \times K)^{(k)} \cong(H \times K)^{(k)}$, and Proposition 2.6 yields

$$
G^{(k)} \times K^{(k)} \cong H^{(k)} \times K^{(k)} .
$$

Proposition 2.1 guarantees that $K^{(k)}$ has a loop at the vertex $\left(x_{0}, x_{1}, x_{2}, \ldots, x_{k-1}\right)$. Corollary 3.3 thus gives $G^{(k)} \cong H^{(k)}$, hence $G \cong H$.

\section{References}

[1] P. Hell and J. Nešetřil, Graphs and Homomorphisms, Oxford Lecture Series in Mathematics, Oxford University Press, Oxford, 2004.

[2] W. Imrich and S. Klavžar, Product Graphs: Structure and recognition, Wiley-Interscience Series in Discrete Mathematics and Optimization, John Wiley and Sons, New York, 2000.

[3] L. Lovász, On the cancellation law among finite relational structures, Period. Math. Hungar. 1(2) (1971), 145-156. 